\title{
HLA and bronchopulmonary dysplasia susceptibility: A pilot study
}

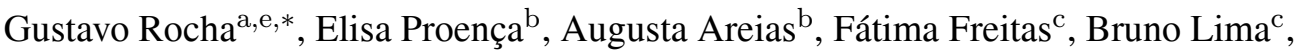 \\ Teresa Rodrigues ${ }^{\mathrm{d}, \mathrm{e}}$, Helena Alves ${ }^{\mathrm{c}}$ and Hercília Guimarães ${ }^{\mathrm{a}, \mathrm{e}}$ \\ ${ }^{a}$ Departament of Pediatrics, Division of Neonatology, Hospital de São João, Porto, Portugal \\ ${ }^{\mathrm{b}}$ Division of Neonatology, Maternity Júlio Dinis, Porto Hospital Centre, Porto, Portugal \\ ${ }^{\mathrm{c}}$ Centre of Histocompatibility of Porto, Porto, Portugal \\ ${ }^{\mathrm{d}}$ Department of Hygiene and Epidemiology, Porto, Portugal \\ ${ }^{\mathrm{e}}$ Faculty of Medicine of Porto University, Porto, Portugal
}

\begin{abstract}
There is little data on the association between Human Leucocyte Antigen (HLA) alleles and Bronchopulmonary Dysplasia (BPD) of the preterm newborn. Our aim was to assess associations between HLA alleles and BPD susceptibility. We studied 156 preterm neonates $(82 \mathrm{M} / 74 \mathrm{~F})<32$ weeks gestational age, alive at 36 weeks gestational age. Detailed clinical data were collected. HLA typing was performed by PCR-SSO. HLA allele frequencies where determined by direct counting for BPD and no-BPD groups. Comparison between BPD and no BPD groups was performed using $t$-test, $\chi 2$ test or Fisher exact test and logistic regression as appropriate. Relative risks (RR) and their 95\% confidence intervals (95\% CI) were also calculated as association measures. We diagnosed $56(35.9 \%)$ neonates with mild BPD and 27 (17\%) with moderate/ severe BPD. We found a significant association between HLA-DRB $1 * 01$ and mild BPD $(\mathrm{OR}=3.48[1.23-10.2])$. The alleles HLA-A*24, $-\mathrm{A} * 68,-\mathrm{B} * 51,-\mathrm{Cw} * 07,-\mathrm{Cw}^{*} 14,-\mathrm{Cw} * 15$ and $-\mathrm{DRB} 1 * 01$ presented a significant association with moderate/ severe BPD. When adjusted to gestational age and birth weight HLA-A*68 $(\mathrm{OR}=5.41[1.46 ; 20.05]),-\mathrm{B} * 51(\mathrm{OR}=3.09[1.11 ; 8.63])$ and $-\mathrm{Cw} * 14(\mathrm{OR}=4.94[1.15 ; 21.25])$ were significantly associated with moderate/ severe BPD. Conclusion - Our findings suggest an association between HLA-A*68, -B*51 and $-C^{*} 14$ and BPD susceptibility, and that an autoimmune mechanism may be implicated in the pathogenesis of the disease.
\end{abstract}

Keywords: HLA, major histocompatibility complex, bronchopulmonary dysplasia, preterm newborn

\section{Introduction}

Chronic lung damage of the preterm newborn is the consequence of disturbed lung development and inflammation due to prematurity and perinatal triggering factors. This condition, generally termed as bronchopulmonary dysplasia (BPD) affects about 20-30\% of very low birth weight infants (birthweight $<1500 \mathrm{~g}$ )

\footnotetext{
* Corresponding author: Gustavo Rocha, Serviço de Neonatologia / Departamento de Pediatria, Hospital de São João - Piso 2, Alameda Prof. Hernâni Monteiro, 4202 - 451 Porto, Portugal. Tel. +351 225512100, extension 1949; Fax: +351 225512273; E-mail: gusrocha@oninet.pt.
}

and is a major cause of morbidity and mortality in this vulnerable population [6,8].

In addition to the infant 's clinical status their genetic predisposition may also contribute to BPD as supported by recent twin studies $[1,9,12]$. Therefore, the association between several polymorphisms and BPD or BPD risk factors is under extensive investigation.

Some studies have directly related the risk of BPD to genome [2]. Fetal inflammatory response syndrome, a major determinant of BPD, is also affected by genotype [2]. Disturbed intrauterine lung development and vascularization may also contribute to BPD; these processes may be impaired in the presence of some rare genetic mutations. Furthermore, there is also a genetic component in the susceptibility to other perina- 
tal adaptational disturbances such as respiratory distress syndrome that are associated with an increased need for mechanical ventilation, and, hence, with lung damage [2].

Little is known about the immunologic mechanisms of the human major histocompatibility complex (MHC), commonly called the human leukocyte antigen (HLA) complex, in the preterm newborn and at which extent it may influence the outcome of certain diseases, as BPD, during the developing process in which the preterm newborn is exposed to various noxious agents.

The development of active autoimmunity appears to require a predisposing genetic background, which means the inheritance of certain haplotypes of the MHC [10]. Some HLA alleles are strongly associated with certain disease states, particularly for some common autoimmune diseases in children and adult populations [11]. Other factors, as infections, inflammation, drugs and chemicals or their metabolic derivates may change the components of the self, originating new antigenic molecules to which a previous tolerance has not been created, and provide the driving force for the activation of an autoimmune response $[4,5,13]$.

The aim of this study was to find an association between MHC alleles and the susceptibility to BPD in preterm neonates.

\section{Material and methods}

Four hundred caucasian preterm neonates less than 32 weeks gestational age (31 weeks and 6 days, inclusive), born between January 2000 and June 2009 at two tertiary neonatal intensive care units (NICU) in the north of Portugal, and alive at 36 weeks postmenstrual age were selected for MHC study. Preterm neonates affected by a TORCH infection, a chromosomal or a major congenital anomaly, twin-twin transfusion syndrome, asphyxia, any inborn error of metabolism detected during the neonatal period, as well as those transferred to another institution or deceased before 36 weeks corrected age, were excluded. Preterm neonates whose parents did not consent, were also not included.

Clinical features, including gender, gestational age, birth weight, antenatal steroid pulses, histological chorioamnionitis, Apgar score, neonatal morbidity, use of exogenous surfactant, oxygen therapy, mechanical ventilation and duration of NICU stay were prospectively collected. The material samples for MHC study were collected before discharge from the NICU or at outpatient consultation, after the discharge.

The study was approved by the institutional research ethics board of the both centres involved in the study. The confidentiality of the collected data was assured and the study was approved by the National Committee for Data Protection.

MHC study: Epithelial cells of oral cavity were collected with appropriate brushes (Citogene ${ }^{\circledR}$ DNA Buccal, Citomed, Portugal). DNA was purified and analysed by PCR/SSO method in Luminex IS100 Langwood Diagnostics equipment (Luminex Corporation, Austin, TX, USA). The classic class I molecules HLAA, -B, -C and class II, HLA-DR were assessed. The analysis was performed at the Histocompatibility Centre of Porto, Portugal.

The diagnosis of bronchopulmonary dysplasia was made according to the NIH (National Institute of Health) Consensus Definition of Bronchopulmonary Dysplasia [12]. In this study we analysed the association between BPD and HLA alleles for those requiring oxygen for 28 days of life and room-air at 36 postmenstrual age (mild BPD) and on supplemental oxygen for 28 days and also on oxygen/positive pressure support at 36 weeks post-menstrual age (moderate/ severe BPD). Oxygen, in both centres, is used to maintain saturations given by pulse oximetry in the range of $88 \%-$ $94 \%$ for respiratory distress syndrome and $90 \%-95 \%$ for bronchopulmonary dysplasia.

Comparison between BPD and no-BPD groups was performed using Chi-square or the Fisher's exact test for categorical variables, or the Mann-Whitney test to compare two independent samples.

HLA allele frequencies were determined by direct counting for neonates with and without BPD. The $\chi^{2}-$ test or Fisher exact test, when appropriate, were used to assess the association of HLA alleles with BPD susceptibility. The $p$ - values were corrected for the multiple comparisons $(\mathrm{pc})$ using the formula $\mathrm{pc}=1-(1$ $-\mathrm{p})^{n}$, with $\mathrm{n}$ the number of alleles at each locus $(n=$ 17 for $\mathrm{A}^{*} ; n=28$ for $\mathrm{B}^{*} ; n=15$ for $\mathrm{C}^{*} ; n=15$ for DRB1*). Relative Risk (R.R.) and their confidence intervals (C.I) at $95 \%$ were calculated to quantify the possible associations. Mann-Whitney test was used to compare mean values of the continuous variables.

Correcting $p$ values for multiple comparisons is common practice in HLA association studies as the loci of this region are very polymorphic and using corrected values ensures that any associations found are not by chance. The alternative to correcting $p$ values for the multiple comparisons is the replication of the results 
with uncorrected $p$-values. This is why we chose to present both the $p$ values corrected for multiple comparisons (pc) and the uncorrected ones (p). Statistically significant uncorrected $p$ values can be considered indicators of future study hypothesis.

Logistic regression models were fit to access HLA alleles association with BPD for adjusted results and Odds Ratio (OR) estimated.

The $p$ values lower than 0.05 were considered statistically significant. All the statistical analysis was performed using the SPSS v13 and EpiInfo software programs.

\section{Results}

One hundred and fifty six preterm neonates were evaluated. The median gestational age and birthweight were 29 (23-31) weeks and 1030 (525-1935) grams, respectively. Fifty six $(35.9 \%)$ neonates were dependent on supplemental oxygen at 28 days of life, 29 (52\%) males, 27 (48\%) females, median gestational age 27 (23-30) weeks, median birthweight 841 (525$1460)$ grams. Twenty seven $(17.3 \%)$ neonates were dependent on supplemental oxygen at 36 weeks postmenstrual age. Demographics and BPD risk factors, considering the dependency on oxygen at 36 weeks of post-menstrual age, are reported in Table 1.

We found an association between HLA-DRB $1 * 01$ and mild BPD ( $n=13$ (23.2\%); OR = 3.48 [1.2310.2]), OR adjusted to birth weight and gestational age. The alleles HLA-A*24, $-\mathrm{A} * 68,-\mathrm{B} * 51,-\mathrm{Cw} * 07$, $-\mathrm{CW}^{*} 14,-\mathrm{CW}^{*} 15$ and DRB1*01 presented an association with moderate/ severe BPD, Table 2 . When adjusted to gestational age and birth weight HLA-A*68, $-\mathrm{B} * 51,-\mathrm{Cw} * 14$ were significantly associated to moderate/ severe BPD, Table 3.

\section{Discussion}

This report characterizes a potential genetic basis for BPD susceptibility in a population of preterm neonates. Several studies have directly related the risk of BPD to genomic variants [2]. Polymorphisms of cytokines (IFN $\gamma \mathrm{T}^{+874} \mathrm{~A}$ ), adhesion molecules (Lselectin-Prot213Ser), elements of rennin-angiotensin system (ACE-I/D), antioxidant enzymes (GST-P1 Val105lle), and surfactant proteins (SPA1, SPBintron4) have been identified as risk factors for BPD [2]. Other studies investigated the role of genotype in
BPD risk factors. Premature birth has been linked to polymorphisms with an impact on immune status (such as IL-6 $\mathrm{G}^{-174} \mathrm{C}$, MBL2 54G/A, VEGF $\mathrm{G}^{+405} \mathrm{C}$, HSP72A ${ }^{+1267}$ Ggenes) and matrix metalloproteases [2]. Fetal inflammatory response syndrome, a major determinant of BPD, is also affected by genotype (including $\mathrm{LT} \alpha \mathrm{A}^{+250} \mathrm{G}$ ). (For a detailed review, see 2$)$.

The human MHC, commonly called HLA complex, is a 4-megabase region on chromosome $6(6 \mathrm{p} 21.3)$ that is densely packed with expressed genes. The best known of these genes are the HLA class I and class II genes, whose products are critical for immunologic specificity and transplantation histocompatibility, and they play a major role in susceptibility to a number of autoimmune diseases. Many other genes in the HLA region are also essential to the innate and antigen-specific functioning of the immune system [11].

BPD is the end result of a pulmonary inflammatory process and lung repair with impaired alveolarization and vascularization in response to a multi-factorial lung injury. BPD is the result of the action of several risk factors over a developing immature lung, which may change the components of the self, originating new antigenic molecules to which a previous tolerance has not been created, and provide the driving force for the activation of an autoimmune response. Other possible mechanisms of this new found association in babies with BPD could be explained by chorioamnionitis and post-natal infection, which will have to be addressed in future studies. Presumably, one or more HLA alleles may be involved in this autoimmune response.

The idea that an autoimmune process might be involved in BPD pathogenesis is relatively novel. Rosen et al. [14] tested the hypothesis that thymic architecture and/or T-cell function is altered with BPD, leading to autoimmunity and immunodeficiency. Their observations suggest that bombesin-like peptides mediate premature thymic maturation and thymic nurse-cell depletion, leading to autoreactive $\mathrm{T}$ cells that could contribute to lung injury.

Mailaparambil et al. [15] also studied 155 infants born with a gestational age $<$ or $=28$ weeks to determine clinical as well as genetic risk factors for the development of BPD in the German population and found that the strongest epidemiological risk factor for BPD was birth weight, followed by low gestational age. Genetic association was detected with single polymorphisms within Tumour necrosis factor alpha, Toll like receptor 10 and vascular endothelial growth factor. The former two genes showed also association with BPD in 
Table 1

Demographics and BPD risk factors, considering the dependency on oxygen at 36 weeks of post-conceptional age $(n=156)$

\begin{tabular}{|c|c|c|c|}
\hline & $\begin{array}{c}\text { BPD present } \\
n=27\end{array}$ & $\begin{array}{c}\text { BPD absent } \\
n=129\end{array}$ & $\mathrm{p}$ \\
\hline Gestational age, median (min - max) weeks & $27(23-30)$ & $29(24-31)$ & $<0.0001$ \\
\hline Birthweight, median (min-max) (g) & $842(630-1460)$ & $1074(525-1935)$ & $<0.0001$ \\
\hline Gender M / F, n (\%) & $\begin{aligned} M & =15(55.6) \\
F & =12(44.4)\end{aligned}$ & $\begin{aligned} M & =67(51.9) \\
F & =62(48.1)\end{aligned}$ & $0.833^{\ddagger}$ \\
\hline Intra-uterine growth restriction, $\mathrm{n}(\%)$ & $4(14.8)$ & $17(13.2)$ & $0.763^{\ddagger}$ \\
\hline \multicolumn{4}{|l|}{ Antenatal steroids, $\mathrm{n}(\%)$} \\
\hline absent & $2(7.4)$ & $8(6.2)$ & \\
\hline incomplete cycle & $7(25.9)$ & $29(22.5)$ & $0.890^{*}$ \\
\hline full cycle & $18(66.7)$ & $92(71.3)$ & \\
\hline Respiratory distress syndrome, $\mathrm{n}(\%)$ & $27(100)$ & $105(81.4)$ & $\mathbf{0 . 0 1 5} 5^{\ddagger}$ \\
\hline Exogenous surfactant & $26(96.3)$ & $84(65.1)$ & $\mathbf{0 . 0 1 1 ^ { \ddagger }}$ \\
\hline Mechanical ventilation, n (\%) & $27(100)$ & $124(96.1)$ & $0.588^{\ddagger}$ \\
\hline Days of ventilation, median (min-max) & $65(1-123)$ & $19(0-105)$ & $<0.0001$ \\
\hline Oxygen, $\mathrm{n}(\%)$ & $27(100)$ & $107(82.9)$ & $\mathbf{0 . 0 1 5}^{\ddagger}$ \\
\hline Oxygen at 28 days, $\mathrm{n}(\%)$ & $24(88.8)$ & $29(22.4)$ & $<\mathbf{0 . 0 1}$ \\
\hline Days of oxygen, median (min-max) & $86(21-365)$ & $3(0-72)$ & $<0.0001$ \\
\hline Patent ductus arteriosus, $\mathrm{n}(\%)$ & $14(51.9)$ & $32(24.8)$ & $\mathbf{0 . 0 0 9} 9^{\ddagger}$ \\
\hline \multicolumn{4}{|l|}{ Nosocomial sepsis, $\mathrm{n}(\%)$} \\
\hline absent & $3(11.1)$ & $44(34.1)$ & \\
\hline 1 episode & $11(40.7)$ & $54(41.9)$ & \\
\hline 2 episodes & 7 (25.9) & $21(16.3)$ & $0.023^{*}$ \\
\hline 3 episodes & $4(14.8)$ & $4(3.1)$ & \\
\hline 4 episodes & $2(7.4)$ & $6(4.7)$ & \\
\hline Histological chorioamnionitis, n (\%) & $12(44.4)$ & $37(28.7)$ & $0.117^{\ddagger}$ \\
\hline Funisitis, n (\%) & $7(25.9)$ & $12(9.3)$ & $\mathbf{0 . 0 2 5 ^ { \ddagger }}$ \\
\hline NICU stay, median (min-max) days & $98(61-149)$ & $61(27-172)$ & $<0.0001$ \\
\hline
\end{tabular}

- Mann-Whitney test; *Pearson Chi-Squared test; ${ }^{\ddagger}$ Fischer’s exact test.

Table 2

HLA allele frequencies in moderate/severe BPD patients and neonates without BPD

\begin{tabular}{|c|c|c|c|c|c|c|}
\hline HLA allele & $\begin{array}{c}\text { BPD present } \\
n=54 \\
\mathrm{n}(\%)\end{array}$ & $\begin{array}{c}\text { BPD absent } \\
n=258 \\
\mathrm{n}(\%)\end{array}$ & $\mathrm{p}$ & $\mathrm{p}_{c}$ & $\mathrm{RR}$ & $95 \% \mathrm{CI}$ \\
\hline$A * 24$ & $1(1.9)$ & $31(12)$ & $0.03 *$ & $\mathrm{~ns}$ & 0.17 & {$[0.02 ; 1.15]$} \\
\hline$A * 68$ & $6(11.1)$ & $10(3.9)$ & $0.04^{\ddagger}$ & ns & 2.31 & {$[1.17 ; 4.58]$} \\
\hline$B * 51$ & $10(18.5)$ & $24(9.3)$ & $0.05 *$ & ns & 1.86 & {$[1.03 ; 3.34]$} \\
\hline $\mathrm{CW}^{*} 07$ & $4(7.4)$ & $54(20.9)$ & $0.02 *$ & $\mathrm{~ns}$ & 0.35 & {$[0.13 ; 0.93]$} \\
\hline $\mathrm{CW}^{*} 14$ & $5(9.3)$ & $6(2.3)$ & $0.03^{\ddagger}$ & ns & 2.79 & {$[1.39 ; 5.60]$} \\
\hline $\mathrm{CW}^{*} 15$ & $7(13)$ & $9(3.5)$ & $0.01^{\ddagger}$ & $\mathrm{ns}$ & 2.76 & {$[1.49 ; 5.09]$} \\
\hline DRB $1 * 01$ & $8(14.8)$ & $14(5.4)$ & $0.03^{\ddagger}$ & ns & 2.29 & {$[1.24 ; 4.23]$} \\
\hline
\end{tabular}

ns - statistically not significant; ${ }^{*} \chi^{2}-$ test; ${ }^{\ddagger}$ Fischer's exact test.

haplotype analyses. The authors concluded that association of BPD was far more convincingly found with a few clinical factors than with genetic polymorphisms. This underscores the genetic complexity of the disease. Furthermore, the identification of predisposing genetic polymorphisms might be hampered by the complex interaction between clinical and genetic factors.

There is a great paucity in the literature regarding to the association between HLA and BPD susceptibility. HLA-A*2 has already been associated with chronic lung disease in preterm neonates less than $1500 \mathrm{~g}$ birth weight, in a single study [3]. This association could not be confirmed in our study. It is also well known that older studies, as the study of Clark et al. [3] could have flaws in the HLA testing and should be carefully considered. To our knowledge, no other studies have corroborated this finding, or the association between BPD and other HLA alleles.

Although prospectively performed over a nine years period, clinical measures and ventilation strategies did not change in both units, and we believe that this issue did not influence the outcome of patients as well as the 
Table 3

Logistic regression analyses for HLA gene differences between moderate/severe BPD patients and neonates without BPD

\begin{tabular}{lccl}
\hline HLA allele & $\mathrm{p}^{*}$ & OR & $95 \% \mathrm{CI}$ \\
\hline $\mathrm{A} * 24$ & $\mathrm{~ns}$ & & \\
$\mathrm{~A} * 68$ & 0.01 & 5.41 & {$[1.46 ; 20.05]$} \\
$\mathrm{B} * 51$ & 0.03 & 3.09 & {$[1.11 ; 8.63]$} \\
$\mathrm{Cw} w^{*} 07$ & $\mathrm{~ns}$ & & \\
$\mathrm{Cw} 14$ & 0.03 & 4.94 & {$[1.15 ; 21.25]$} \\
$\mathrm{Cw} 15$ & $\mathrm{~ns}$ & & \\
$\mathrm{DRB} 1 * 01$ & $\mathrm{~ns}$ & & \\
\hline
\end{tabular}

ns - statistically not significant; *adjusted $p$-values for gestational age and birth weight.

results of the study.

In our pilot study, HLA-A*68, -B*51 and $-\mathrm{CW}^{*} 14$ were the HLA alleles associated with oxygen requirement at 36 weeks post-menstrual age in preterm neonates less than 32 weeks gestational age. These HLA alleles have never been described to be associated to any other diseases or clinical conditions in newborns, infants or adults. It should be emphasized that the finding of a genotype-disease association does not demonstrate a causative relationship, and this should be addressed in future studies with larger samples. Further studies are also needed to corroborate our findings, and to detect other genetic variants predisposing to BPD. This would allow the identification of patients at risk for lung damage that might benefit from any future specific therapeutic intervention as well as from caffeine beyond treatment of apnoea and intra-muscular vitamin A. By now, our findings need to be interpreted in the context of this study only and cannot be generalized in wider context.

\section{Acknowlegments}

To Alexandra Almeida, MD, from Maternity Júlio Dinis, for the collaboration given in samples collection, and to Sandra Tafulo, from the Histocompatibility Centre of Porto, for the help given in the laboratory processing of the DNA and HLA analysis.
This study was partially supported by Zeru Award 2008 of the Portuguese Society of Neonatology, granted by Respisense ${ }^{\circledR}$.

\section{References}

[1] V. Bhandari, M.J. Bizzarro, A. Shetty et al., for the Neonatal Genetics Study Group. Familial and genetic susceptibility to major neonatal morbidities in preterm twins, Pediatrics 117 (2006), 1901-1906.

[2] G. Bokodi, A. Treszl, L. Kovács et al., Dysplasia: A review, Pediatr Pulmonol 42 (2007), 952-61.

[3] D.A. Clark, L.G. Pincus, M. Oliphant et al., HLA-A2 and chronic lung disease in neonates, J Am Med Assoc 248 (1982), 1868-1869.

[4] U. Christen and M.G. von Herrath, Induction, acceleration or prevention of autoimmunity by molecular mimicry, Mol Immunol 40 (2004), 1113-1120.

[5] U. Christen and M.G. von Herrath, Infections and autoimmunity - good or bad? J Immunol 174 (2005), 7481-7486.

[6] H. Christou and D. Brodsky, Lung injury and bronchopulmonary dysplasia in newborn infants, $J$ Intensive Care Med 20 (2005), 76-87.

[7] A. Jobe and E. Bancalari, NICHD/NHLBI/ORD Workshop Summary - Bronchopulmonary Dysplasia, Am J Respir Crit Care Med 163 (2001), 1723-1729.

[8] J.P. Kinsella, A. Greenough and S.H. Abman, Bronchopulmonary dysplasia, Lancet 367 (2006), 1421-1431.

[9] P.M. Lavoie, C. Pham and K.L. Jang, Heritability of bronchopulmonary dysplasia, defined according to the Consensus Statement of the National Institutes of Health, Pediatrics 122 (2008), 479-485.

[10] H. MacDonald, American Academy of Pediatrics. Committee on Fetus and Newborn Perinatal Care at the Threshold of Viability, Pediatrics 110 (2002), 1024-1027.

[11] G.T. Nepom, The major histocompatibility complex, in: Harrison's Principles of Internal Medicine, (17th ed.), A.S. Fauci, E. Braunwald, D.L. Kasper, S.L. Hauser, D.L. Longo, J.L. Janeson and J. Loscalzo, eds, New York, The McGraw-Hill Companies, Inc; 2008, 2045-2053.

[12] L.A. Parton, S.S. Strassberg, D. Qian et al., The genetic basis for bronchopulmonary dysplasia, Front Biosci 11 (2006), 1854-1860.

[13] W.J. Pichler, Drug-induced autoimmunity, Curr Opin Allergy Clin Immunol 3 (2003), 249-253.

[14] D. Rosen, J.H. Lee, F. Cuttitta et al., Accelerated thymic maturation and autoreactive T cells in bronchopulmonary dysplasia, Am J Respir Crit Care Med 174 (2010), 75-83.

[15] B. Mailaparambil, M. Krueger, U. Heizmann et al., Genetic and epidemiological risk factors in the development of bronchopulmonary dysplasia, Dis Markers 29 (2010), 1-9. 


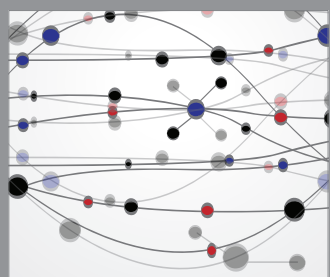

The Scientific World Journal
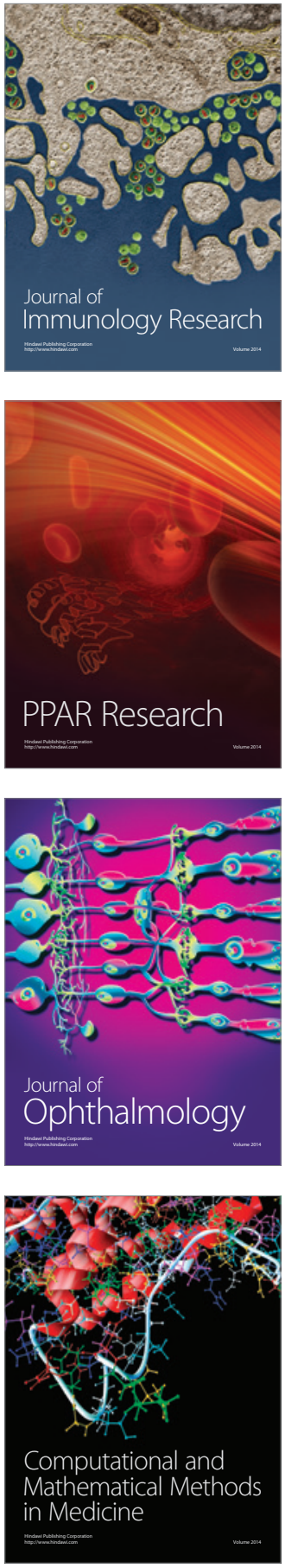

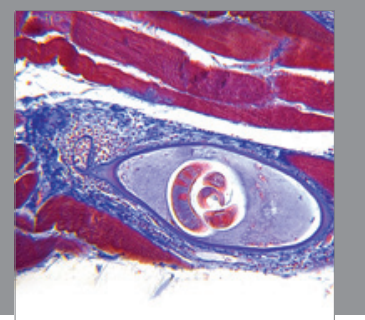

Gastroenterology

Research and Practice
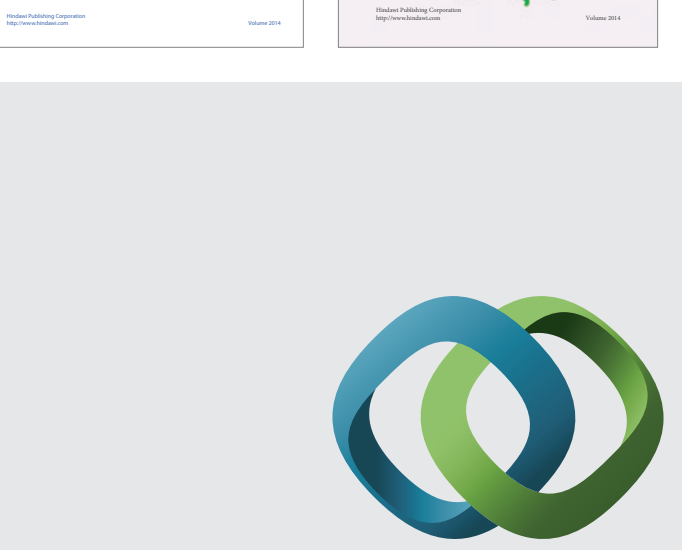

\section{Hindawi}

Submit your manuscripts at

http://www.hindawi.com
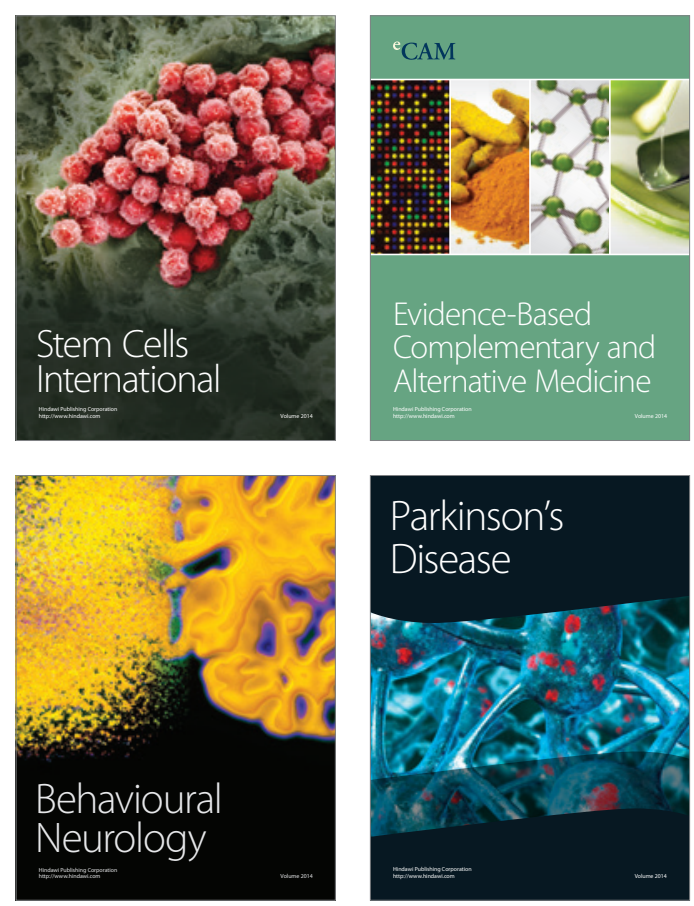

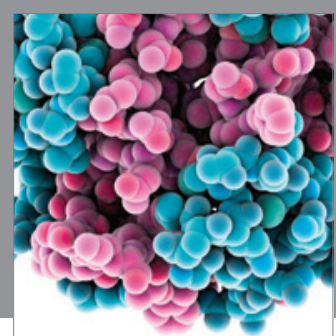

Journal of
Diabetes Research

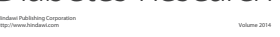

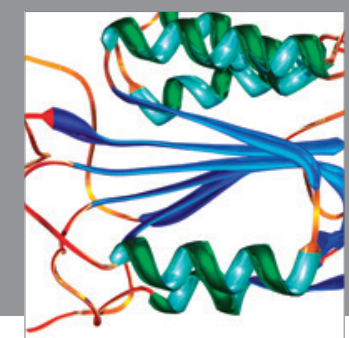

Disease Markers
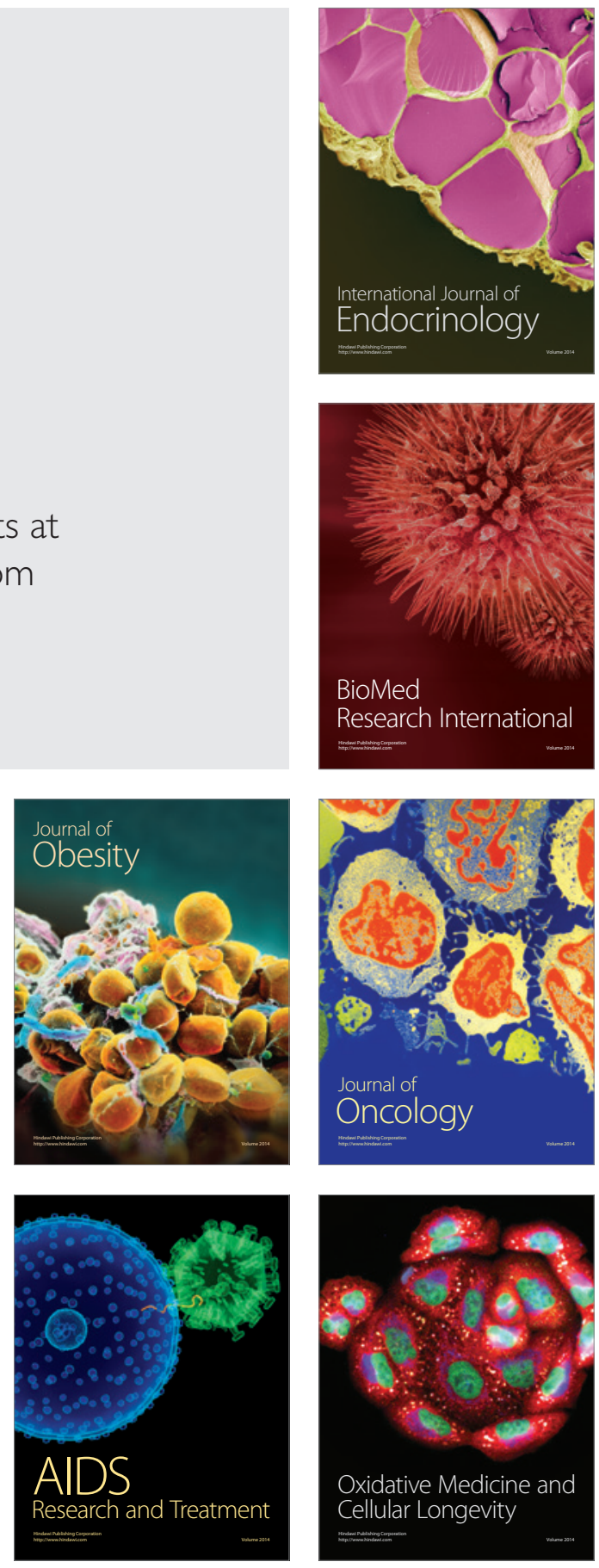